\title{
Midwives' and Medical professionals' perspectives of collaborative practice at Queen Elizabeth Central Hospital Maternity Unit, Malawi: The discovery phase of an appreciative inquiry project
}

\author{
Elizabeth Chodzaza ${ }^{1}$, Christina Mbiza ${ }^{2}$, Luis Gadama ${ }^{3}$, Ursula Kafulafula ${ }^{1}$ \\ 1. University of Malawi Kamuzu College of Nursing, Faculty of Midwifery, Neonatal and Reproductive Health Studies, Blantyre, Malawi \\ 2. Queen Elizabeth Central Hospital, Department of Obstetrics and Gynecology, Blantyre, Malawi \\ 3. University of Malawi, College of Medicine, School of Medicine, Blantyre, Malawi
}

\section{Background \\ Abstract}

This paper reports on part of a larger study, the aim of which was to develop an intervention to collaboratively develop innovative strategies to promote effective collaborative practices among midwives and medical professionals working in intrapartum care unit. Collaborative practice is a critical marker for success in improving quality of maternity care. To date, there has been limited exploration of collaborative practices between midwives and medical professionals working in intrapartum care from the African perspective.

Aim

This paper reports findings of the discovery phase of appreciative inquiry (AI) set out to understand the perspectives of midwives and medical professionals on collaborative practices at Queen Elizabeth Central Hospital labour and delivery ward in Malawi.

Methods

The study used an exploratory qualitative approach framed in an Appreciative Inquiry theoretical perspective. Appreciative Inquiry consists of four phases :(discovery, dream, design and destiny).

The discovery phase consisted of 16 in-depth interviews and 2 focus group discussions among purposively selected midwives $(4$ nurse midwives, 2 midwifery unit matrons) and medical professionals (2 obstetricians, 4 registrars, 2 intern doctors, 2 clinical officers) working in the labour ward. All interviews and discussions were audiotaped and transcribed verbatim. Data were analysed using thematic analysis. Results: Five dominant themes emerged: collaborative breakdown, benefits of collaboration, the importance of positive and respectful attitude, barriers to effective collaborative practices and strategies to improve collaborative practice.

Conclusion/Recommendations

Aligning the perspectives of the members of the two disciplines is significant to effective implementation of collaborative intrapartum care. Participants demonstrated that there is increased parallel working of midwives and doctors at QECH. This is not professionally healthy. Therefore, putting together the viewpoints of the professions to create a mutually agreeable professional framework of collaborative intrapartum practice is significant. Additionally, there is an obvious need to address the professional concerns of both disciplines.

Keywords: Midwives, Medical Professionals

\section{Introduction}

Optimum collaboration between maternity service providers of different professions in a labour ward is a critical element of safe and quality care. Studies on maternity care services have revealed that lack of collaboration among care providers is usually associated with poor outcomes ${ }^{1}$. Maternal mortality remains one of the biggest challenges to public health in economically less developed countries (ELDCs) ${ }^{2,3,4}$.

Childbirth in Malawi is generally unsafe for the majority of women and their babies. Problems in maternity care persist and disproportionately affect women and babies ${ }^{5,6}$. Malawi's maternal mortality ratio (MMR), currently estimated at 439/100,000 live births ${ }^{7}$, is still high. The country remains far from meeting its commitment to Sustainable Development Goal (SDG) No.3, which is to reduce maternal mortality to 70 per 100,000 births $^{4,8,9}$. Studies on causes of maternal mortality and morbidity have indicated that maternity care during labour has not been of a sufficient standard to improve the outcomes for women giving birth 5,6. Kirkup ${ }^{10}$ argues that positive collaborative practices between midwives and obstetrical care providers is necessary for provision of quality maternity care. Global evidence has shown that professional relationships in maternity care have a large influence on midwifery practice and subsequent clinical decision making ${ }^{11,12}$. Kirkup ${ }^{10}$ reported substandard clinical competence, with deficient skills and knowledge, poor collaborative practices between different staff groups as factors that lead to unnecessary deaths of mothers and babies ${ }^{10}$.

Anecdotal reports and a few studies in Malawi indicate a lack of collaborative practice between midwives and other obstetrical care providers ${ }^{13,14}$. This might partly explain the high MMR in Malawi.

One problem is the lack of studies on collaborative practices among midwives and medical professionals working in delivery suites. Specifically, our literature review revealed that systematic data on Africa, and Malawi in particular, are largely absent. The limited data available on collaborative practices are from western countries. This makes Malawi fertile ground for further research. The aim of this study was to gain an in- 
depth understanding of the perspectives of midwives and medical professionals who work on collaborative practice in one delivery suite.

\section{Methodology Study design}

We employed an exploratory qualitative design that allowed us to explore a wide range of issues in relation to certain social phenomena. The approach allowed greater understanding of group dynamics and inherent anxieties that pervade the midwifery and medical professions during provision of intrapartum care ${ }^{15,16}$. The research involved a variety of professions, where each had different knowledge depending on their position and experience. This situation was ripe for a collaborative effort to find best practices and enforce positive change.

The study was framed in an Appreciative Inquiry (AI) theoretical research perspective. An AI approach offers a participatory framework that promotes the concept that social groups naturally construct their reality; and also, they can discover what is good and true ${ }^{17}$. AI uses a cyclical process that consists of four phases: (discovery, dream, design and destiny). The discovery phase is the first and critical stage of the inquiry. It involves collection of useful data ${ }^{17}$. This paper reports the findings of the discovery phase of what we hope will be a four-phase AI.

\section{Study setting}

The study was conducted at Queen Elizabeth Central Hospital $(\mathrm{QECH})$, a public tertiary hospital located in Blantyre, Malawi. The facility provides specialist medical, obstetric and gynaecological services, and has a bed capacity of 1,100. The maternity unit has 250 beds for both gynaecological and obstetric patients; 35 beds are for labour and delivery. The unit is a teaching facility for health professionals from various colleges in Malawi. The labour ward has a total of 21 midwives, with an average of 6-8 midwives allocated per shift. There are 3 Senior Nursing officers for the unit, 10 Obstetricians and Gynaecologists, 12 Registrars and 25 Doctors on internship. The MMR, according to Health Information System 2017/18, was 632 deaths per 100,000 live births, and the perinatal mortality rate was 86 per 1000 births ${ }^{18}$.

\section{Population and Sample}

We purposively selected 16 participants working in the labour and delivery ward: four nurse midwives, two midwifery unit matrons, two obstetricians, four registrars, two intern doctors and two clinical officers. This purposive sampling technique allowed a diversity of perspectives from the health care professionals, which provided the researchers with the critical information ${ }^{19,20}$. Specifically, we used a pragmatic approach of maximum variation sampling strategy by selecting a small but diverse sample of participants ${ }^{19}$. These individuals were able to offer a wide variety of perspectives related to collaborative practices in intrapartum care. The first author made a presentation to potential participants to explain principles of the study in more detail. An information package, which included details about the study, an invitation to participate, and consent forms, was given to potential participants for them to read the information. We followed up after two weeks of their reading through the research details. We explained that we were choosing specific midwives and medical professionals to enhance variation within the sample. Together, we reviewed the principles of the study in more detail. None of the midwives or medical professionals we approached declined to participate in the study.

\section{Data collection and analysis}

Data were collected through individual face-to-face in-depth interviews and focus group discussions (FGD). A semistructured guide in English was used to aid data collection. After conducting a literature review, we developed semistructured interview questions to guide the interviews. We used the AI model for the discovery phase to develop these questions.

Individual face-to-face interviews lasting 45-60 minutes were conducted to explore accounts of the participants' collaborative practice perspectives. The participants decided on the place and time for their interviews. The researchers maintained reflective diaries, wherein they documented their interpretations of interactions between the participants and themselves. All interviews were interdisciplinary, meaning that researchers interviewed only participants of other professions. This was done to reduce bias within any one profession.

We conducted two FGDs, each including seven participants, one from each of the seven different professions. We explored issues that came out of the earlier individual interviews. Both in the individual interviews and in the FGDs the focus was on each participant's experience with and attitudes towards collaborative practice in the labour ward. A digital recorder was used to record interviews and FGDs. The interviews and FGDs were transferred onto a word processor immediately after each event. All data were entered into a personal computer with a security code for access.

We adapted Braun and Clarke's (2006) thematic analysis to analyse data ${ }^{21}$. Data were analysed manually and this allowed the data to be coded inductively and deductively in order to gather information related to perspectives on collaborative practices $^{22,23}$. All interviews were transcribed verbatim. The transcriptions were read to identify important words, phrases and statements of information in relation to the research topic. The selected information constituted meaningful units and aspects related to each other by content and context. Codes were assigned to meaningful units. Similar codes were aggregated into categories and then developed into themes through a method of abstraction ${ }^{24}$. Peer debriefing was done with all researchers throughout the research process. This provided a second view of the quality and content of data collected and themes generated. The insights were incorporated during data analysis. During debriefing meetings, we gained feedback and new ideas about what meaning could be drawn from the findings. This process helped to correct for bias and address other problems in data interpretation.

\section{Ethical considerations}

The study was approved by the Institutional Review Board of College of Medicine Research Ethics Committee (Certificate No P.04/17/2159). Appropriate permission was obtained from all gate keepers. Written informed consent was obtained from all participants. Participants were assured of anonymity and confidentiality and were told they could voluntarily withdraw at any point in the study. A 
full participant information sheet was initially given to each potential participant, who then signed a consent form after deciding to participate in the study.

\section{Findings}

\section{Demographic data of participants}

The sixteen professional participants, aged 22 to 50, consisted of: two registered midwives, two nurse midwife technicians, two midwifery unit matrons, two obstetricians, four registrars, two intern doctors and two clinical officers. The participants had worked in the intrapartum care unit for periods ranging from three months to fifteen years.

\section{Themes emerging from the study}

Five themes emerged that describe the perspectives of collaborative practice of midwives and other medical professionals working in intrapartum care: (1) collaborative breakdown; (2) benefits of collaboration; (3) the importance of a positive and respectful attitude; (4) barriers to effective collaborative practice; and (5) strategies to improve interprofessional collaborative practice. These themes are discussed below with direct quotations from the participants included. All names used in the extracts are pseudonyms.

\section{Collaborative Breakdown}

There was a general consensus among participants on the meaning of collaborative practice and all expressed increased understanding of the value of working collaboratively.

"Collaboration means different cadres working together to provide effective care to women in labour." (Edna)

"Different cadres: midwives, doctors including ward attendants working together to achieve positive maternal and neonatal outcomes." (Natasha)

In principle, findings reveal that doctors and midwives agree on the concept of collaborative practice. However, further analysis showed that there is a difference between espoused beliefs and actual practice.

"I know that there isn't good collaboration between midwives and medical professionals. We work in parallel." (Georgina)

"Currently, midwives and medical professionals work alongside one another, and do not technically collaborate." (Ashley)

Participants expressed that historically, collaboration between midwives and medical professionals worked well, unlike currently.

"There is a disconnect between midwives and doctors now. When I was on internship, collaboration worked quite well." (Wanangwa)

Additionally, participants suggested that the introduction of postgraduate training in the medical college has perpetuated a disconnection between midwives and doctors, where doctors on internship are no longer taught by midwives.

"When I was an intern, collaboration between doctors and midwives worked quite well. This time we have a postgraduate training programme and intern doctors are taught by postgraduate students; doctors no longer think that midwives are as valuable as they used to be when they were teaching intern doctors." (Nema)

Participants noted that doctors are the leaders in the labour ward, and this has affected autonomous midwifery practice in intrapartum care.

"Registrars have taken over leadership in the labour ward. When I was an intern doctor, I relied more on midwives." (Nema)

\section{Benefits of multidisciplinary collaborative intrapartum care}

Participants mentioned numerous benefits of practicing collaboratively, both to women and care providers.

\section{Mechanism for ongoing communication}

Communication was identified as being significant in improving the safety of women in labour.

"I experienced one incident where I told a midwife that we should give a woman in labour some time to push to give birth. However, the midwife told me that the baby will not come out.' So, I went back to re-examine the woman and actually found features of cephalo-pelvic disproportion were prominent. Therefore, we ended up with a good outcome for this woman after performing a caesarean section." (Mercifine)

\section{Optimizes participation in decision making within and across disciplines}

In circumstances where the medical professionals worked together with midwives, participants discussed in a positive way the importance of creating opportunities for interactions regarding care of women in labour.

"We used to work as a team where a medical team would come for a ward round and then a midwife could present a woman she is caring for and then discuss progress of labour and make a decision on care for the woman as a team." (Adrian)

Midwives expressed appreciation of being able to contribute their views and decisions on the care of women in labour.

"I really appreciated when I was given time to present my client and then we were able to discuss care provided and, together as a team, made a decision on next course of action for our client." (Pamela)

The analysis has further revealed that midwives today typically lack assertiveness in the care of women in labour.

"T really wish midwives working in labour ward were more assertive in the way they provide care to women. They should be present during ward rounds so that there are collaborative discussions and team work." (Brandina)

Participants expressed that the medical professionals must provide an enabling environment, where midwives can fully utilize their professional knowledge and contribute effectively in the care of women in labour.

"I remember we had these particular consultants who were not just giving instructions during ward rounds, but would ask midwives' suggestions about how the woman under review should be cared for. This approach made everyone flexible to participate in collaborative care." (Thoko)

\section{The importance of positive and respectful attitude}

The importance of professional conduct, a positive attitude, respect, and clear communication were supported by all participants.

"I think working together with the midwives and asking questions where necessary even when I said something that is not practical, I would respect their views because I am not on the ward 24 hours, but they are. It's a matter of respecting other people's views!" (Mercifine)

Another participant commented about the importance of having a positive attitude and being respectful as a way of mitigating negative working relationships.

"I think the first important thing is to have positive attitude. You need to start your day with a smile and when interacting with colleagues make sure you are not rude and don't show disinterest; then our relationships would be great." (Valera)

Another participant spoke about the need to improve consulting relationships, and thus the care of women in https://doi.org/10.4314/mmj.v32i1.4 
labour.

'When relationships are good, it's nice and motivating to work in such environment. It would be very easy for people to consult each other during care of women in labour." (Eddie)

\section{Barriers to effective collaboration}

\section{Hectic intrapartum care environment}

Participants spoke of the hectic environment of the labour ward and how their stress and frustration impacted collaborative relationships.

"Our working environment is quite depressing; we might think these people don't want to cooperate, but it's so busy, it's hectic, so I feel that contributes to the stresses and fuels up everything." (Nema)

\section{Shortage of midwifery staffing}

Shortage of midwifery staffing was considered a barrier to collaboration and acted as a source of conflict between midwives and doctors.

"I think collaboration is very difficult because sometimes instead of you being physically at the ward round to discuss issues pertaining to women's care, you are running up and down looking after several women in labour; this provokes conflict between you and the doctor."(Adrian)

Due to the shortage of midwifery staffing, midwives from other departments work in labour ward as 'locum' midwives. However, these midwives are not well versed in routines and administration of the labour ward.

"You find that a usual midwife is there today and on other days you work with midwives who are on locum. These people are just new, they don't know how to run the labour ward. Things can work very well if there was sufficient number of midwives consistently working on labour ward." (Roy)

Shortage of material resources affected collaborative practice as midwives had to move between wards to find resources for the care of women in labour.

"You find yourself busy looking for resources to use during care of women in labour and you have no time to collaborate." (Natasha)

\section{Strategies to facilitate collaboration}

\section{Effective Communication}

Effective communication in the labour ward was discussed as a way of improving collaborative practices.

"I need to communicate effectively all the time. The communication can be verbal where the person is right there, or we use medical records in form of writing which should be clear" (Edna)

\section{Continuous Professional Development (CPD) sessions}

A number of participants mentioned the importance of updating knowledge through in-service education across disciplines, which could also increase collaboration.

'I recommend reintroduction of CPD sessions which CoM used to conduct for both midwives and doctors. These need to restart for people to continue updating their knowledge. In addition to obstetric and midwifery skills, we can have CPDs on collaboration itself." (Roy)

\section{(c)Understanding Each Other's Roles}

Many participants discussed the importance of mutual understanding of each other's roles.

"We need to respect roles of each profession; this would include collaborative roles. Therefore, we should be respecting input from every individual then people will see that everyone is valued and there will be sense of shared responsibility." (Clinical Officer 1)

\section{(d) Teamwork}

Participants reported that teamwork helps improve communication and the care that is given to a woman.

"If we are to work collaboratively, we need to communicate and work as a team. This is helpful because one cannot do everything by oneself since it's a multidisciplinary approach to care." (Ashley)

\section{(e)Guidelines to promote collaborative practice}

Participants described positive aspects of having guidelines. They spoke about guidelines promoting collaboration, creating common ground, establishing expectations amongst clinicians who work together and ensuring everybody's speaking the same language.

"I think guidelines promote collaboration because they are common ground that we can all agree and speak same language." (Adrian)

"We need to come up with guidelines that will guide our operations in labour ward. For example, come up with expectations during consultant ward round where we could prescribe that midwives should be in attendance." (Eddie)

\section{Discussion}

The discovery phase of AI is what this study focused on. This phase involved collection of useful data where participants reflected on and discussed collaborative practice in labour ward. The study has demonstrated that collaborative practice allows multiple health workers from different professional backgrounds to work together with clients to deliver the highest quality of care and achieve local health goals. This matches what others have found, namely: that health care providers should work in "true partnership" in order to accomplish tasks that no individual can achieve alone ${ }^{25,26}$. All participants concurred on the meaning of collaboration. Agreement on a commonly acceptable definition from perspectives of both midwives and medical professionals is essential for successful implementation of collaborative care initiatives. This common understanding assists different professionals to speak the same language and have the will to clarify their roles, build trust and develop respect for different viewpoints in intrapartum care.

Although midwives and medical professionals agreed on the definition of collaborative practice, in general, analysis revealed a marked difference in the application of the definition during provision of intrapartum care. This finding is critical because it indicates that the two professions are not communicating well enough. Unfortunately, participants did not include the client in their definitions of collaborative practice. This clearly needs to change as we move forward and all professions see the client as integrally involved in her own care. Indeed, none of the participants provided detailed discussion on how to integrate clients into the care team despite the fact that clients are recognized as the ultimate justification for collaborative care. However, working in positive relationship with clients is considered one of the basic tenets of the new midwifery ${ }^{27}$. A labouring woman is an active partner in decision-making for her own care. Midwives and doctors in a study conducted by Hastie and Fahy confirmed that positive interactions are collaborative and include the woman and her partner ${ }^{28}$.

Our findings have demonstrated that midwives and medical 
professionals perceive collaborative practice as beneficial to both clients and care providers. These findings are comparable with those in other studies which have demonstrated that positive interactions are collaborative and are associated with best possible outcomes and experiences ${ }^{29}$. Similarly, poor communication has been identified as the most common cause of preventable unfavourable outcomes ${ }^{30,31,32}$.

Teamwork was identified as a strategy to improve collaborative practices. At the practical level, it meant staff being helpful and pulling together as a team, taking responsibility and initiative, but also being a team player. Participants acknowledged the need to respect each other not just as individuals but professionally. The concept of "professional courtesy" needs to form the basis of routine ward policy. Additionally, there is a need for a deliberate move to a democratic structure of shared, mutual respect and commitment to working in partnership.

Despite differences in their ideals, participants in this study sought mutual trust. Unfortunately, effective teamwork is currently limited by tensions over role boundaries and distribution of power, which are intensified by increasing workloads and a fragmented labour force. Our study shows that maternity care providers aspire to collaborate despite some differences in their understandings of what collaborative care means. Participants in our study have suggested developing guidelines that will promote collaboration, create common ground, and establish shared expectations amongst clinicians who work together. Each profession's scope of practice would consider the respective philosophies and levels of different expertise each maternity care profession brings to a woman's care. This would assist the medical professionals and midwives to recognise their specific areas of expertise, each of which might be required at different times according to a woman's needs. There are several institutional factors that can shape the way a team of people work collaboratively, promoting co-operation instead of division. For example, our study identified shortage of midwifery staffing as a major barrier to effective collaboration. The shortage perpetuates lack of collaboration as midwives lack time for collaboration. Also, the necessary reliance on locum staff means they have insufficient knowledge and experience to collaborate. Collaborative practice has been identified as a promising means of strengthening health systems and improving health outcomes. Such collaboration is increasingly regarded as important for health systems to meet complex health needs given the limited human and financial resources ${ }^{33,34}$.

\section{Conclusion}

Aligning the perspectives of the members of the two disciplines is significant to effective implementation of collaborative intrapartum care. Participants demonstrated that there is increased parallel working of midwives and doctors at QECH. This is not professionally healthy. Therefore, putting together the viewpoints of the professions to create a mutually agreeable professional framework of collaborative intrapartum practice is very crucial. Additionally, there is an obvious need to address the professional concerns of both disciplines.

\section{Strengths and Limitations of the study}

Midwives, registrars, matrons, intern doctors and consultants were included in the study which allowed the researchers to have diverse views of the perspectives on collaborative practices. This research has helped to clarify professional roles surrounding the care of women in labour and existing constraints regarding the input of midwives' and the medical professionals' knowledge, experience and skills towards care of women in labour. However, the study was conducted at one tertiary hospital, as such the results may not be generalised to other settings. However, there is a possibility of transferring the findings to comparable situations.

\section{Disclosure Statement}

The authors declare that they have no competing interests

\section{Funding}

This research was supported by the Norwegian Programme for Capacity Development in Higher Education and Research for Development under the NORHED - MWI-13/0032.

\section{Authors' contributions}

The first author (EC) developed the study design, interview and focus group discussion guides, analysed the data and drafted the manuscript. The co-authors (UK, LG and CM) participated in conducting interviews and focus group discussions, supervised the overall data collection and management, and maintained the data files. All authors contributed to the manuscript writing, they all read and approved the final manuscript.

\section{References}

1. Simpson KR, James DC, Knox GE. Nurse-physician communication during labor and birth: Implications for patient safety. J Obstet Gynecol Neonatal Nurs. 2006 Jul 1;35(4):547-56.

2. Health in 2015: from MDGs, Millennium Development Goals to SDGs, Sustainable Development Goals. Geneva: World Health Organization; 2015.

3. Roos N, von Xylander SR. Why do maternal and newborn deaths continue to occur? Best Pract Res Clin Obstet Gynaecol. 2016 Oct 1; 36:30-44.

4. Nyfløt L, Sitras V. Strategies to reduce global maternal mortality. Acta Obstet Gynecol Scand. 2018 Jun;97(6):639-40.

5. Chodzaza E, Bultemeier K. Service providers' perception of the quality of emergency. Malawi Med J. 2010 Dec;22(4):104-11.

obsteric care provided and factors indentified which affect the provision of quality care. Malawi Med J. 2010;22(4).

6. Kumbani CL, Sundby J, Odland OJ. Factors associated with perinatal deaths in women delivering in a health facility in Malawi. J Nurs Midwifery Res. 2012; 1:69-79.

7. Malawi demographic and health survey 2010. Zomba: National Statistical Office.

8. Ministry of Finance, Economic, Planning and Development. 2014 Millennium Development Goal Report For Malawi. Lilongwe: Mallawi Ministry of Finance and Economic Planning; 2014

9.United Nations Development Programme (UNDP). Malawi millennium development goals endlinereport.2011. Available from: https://www.undp.org/content/dam/malawi/docs/general/UNDP_MW_ EDP_MDG_book final.

10. Kirkup B. The Report of the Morecambe Bay Investigation: An independent investigation into the management, delivery and outcomes of care provided by the maternity and neonatal services at the University Hospitals of Morecambe Bay NHS Foundation Trust from January 2004 to June 2013. Stationery Office; 2015. 
11. Sleutel M, Schultz S, Wyble K. Nurses' views of factors that help and hinder their intrapartum care. J Obstet Gynecol Neonatal Nurs. 2007 May;36(3):203-11.

12. Keating A, Fleming VE. Midwives' experiences of facilitating normal birth in an obstetric-led unit: a feminist perspective. Midwifery. 2009 Oct 1;25(5):518-27.

13. O’Donnell E, Utz B, Khonje D, Van Den Broek N. 'At the right time, in the right way, with the right resources': perceptions of the quality of care provided during childbirth in Malawi. BMC Pregnancy Childbirth. 2014 Dec;14(1):248.

14. Chodzaza E. Midwifery Decision Making During the First Stage of Labour within the Malawian Context. (dissertation). University of Edinburgh; 2016.

15. Saks M, Allsop J. (2007). Researching Health: Qualitative, Quantitative and Mixed Methods. London: Sage Publications.

17. Trajkovski S, Schmied V, Vickers M, Jackson D. Using appreciative inquiry to transform health care. Contemporary nurse. 2013 Aug 1;45(1):95-100.

18. Queen Elizabeth Central Hospital. Health Management Information System. 2017/2018.

19. Patton MQ, CA: Sage. Qualitative Research and Evaluation Methods: Intergrating Theory and Practice. 4th ed. Thousand Oaks: Sage Publications; 2015.

20. Stringer, E, Dwyer, R. Action Research in Human Services.1st ed. Alexandria.VA: Prentice Hall; 2005.

21. Braun V, Clarke V. Using thematic analysis in psychology. Qualitative research in psychology. 2006 Jan 1;3(2):77-101.

22.Boyatzis RE. Transforming qualitative information: Thematic analysis and code development. Thousand Oaks: Sage Publications; 1998.

23.Creswell JW, Klassen AC, Plano Clark VL, Smith KC. Best practices for mixed methods research in the health sciences. Bethesda (Maryland): National Institutes of Health; 2011.

24. Vaismoradi M, Jones J, Turunen H, Snelgrove S. Theme development in qualitative content analysis and thematic analysis. J Nurs Educ Pract [Internet]. 2016 Jan 15 [cited 2020 Apr 3];6(5):p100.

25. Hopkins D. Framework for Action on Interprofessional Education
\& Collaborative Practice. Genva: World Health Organization; 2010.

26. Behruzi R, Klam S, Dehertog M, Jimenez V, Hatem M. Understanding factors affecting collaboration between midwives and other health care professionals in a birth center and its affiliated Quebec hospital: a case study. BMC Pregnancy Childbirth. 2017 Dec;17(1):200.

27. The International Confederation of Midwives [Internet].The Confederation; 2018 [cited 2020, March 28]. The International Confederation of Midwives ; [ICM international definition of the midwife]. Available from https://www.internationalmidwives.org/ourwork/policy-and-practice/icm-definitions.html

28. Hastie C, Fahy K. Inter-professional collaboration in delivery suite: a qualitative study. Women Birth. 2011 Jun 1;24(2):72-9.

29. Berridge EJ, Mackintosh NJ, Freeth DS. Supporting patient safety: examining communication within delivery suite teams through contrasting approaches to research observation. Midwifery. 2010 Oct 1;26(5):512-9.

30. Waldman R, Kennedy HP, Kendig S. Collaboration in maternity care: possibilities and challenges. Obstet Gynecol Clin North Am. 2012 Sep 1;39(3):435-44.

31. Posthumus AG, Schölmerich VL, Waelput AJ, Vos AA, De JongPotjer LC, Bakker R, Bonsel GJ, Groenewegen P, Steegers EA, Denktaş $\mathrm{S}$. Bridging between professionals in perinatal care: towards shared care in the Netherlands. Matern Child Health J. 2013 Dec 1;17(10):1981-9.

32. Downe S, Finlayson K, Fleming A. Creating a collaborative culture in maternity care. J Midwifery Womens Health. 2010 May 6;55(3):2504 .

33. Mickan S, Hoffman SJ, Nasmith L, World Health Organization Study Group on Interprofessional Education and Collaborative Practice. Collaborative practice in a global health context: Common themes from developed and developing countries. J Interprof Care. 2010 Sep $1 ; 24(5): 492-502$.

34. Reeves S, Perrier L, Goldman J, Freeth D, Zwarenstein M. Interprofessional education: effects on professional practice and healthcare outcomes. Cochrane Database of systematic reviews. 2013(3). 